\title{
DISCOVERY OF LIGHT VARIABILITY \\ IN THE WHITE DWARF G 29-38
}

\author{
O.S.SHULOV \\ Astronomical Observatory of Leningrad State University, U.S.S.R.
}

\begin{abstract}
Rapid variations with small amplitudes were detected in the light of the white dwarf EG $159=$ G 29-38, a 13th magnitude star.
\end{abstract}

Before October 1973, three white dwarfs from the list of Eggen and Greenstein (Eggen and Greenstein, 1965) were known to exhibit the rapid light variations with small amplitudes: HL Tau-76, G 44-32, and R 548. Data on these stars were summarized by Lasker and Hesser (1971) in Table 2 of their paper. The fourth star discussed in that paper, $\mathrm{HZ} 29=\mathrm{AM} \mathrm{CVn}$, was proved to be an ultra-short period eclipsing system (Faulkner et al., 1972) and, therefore, must be excluded from this group because the three stars mentioned above did not seem to be members of close binaries.

We discovered another variable white dwarf of this type, EG $159=$ G 29-38, and performed a preliminary photometric study of it during four nights in October-November, 1973. We refer to Shulov and Kopatskaya (1974a, b) for details of the observations. In unfiltered light the variations in brightness are well above the statistical errors and, therefore, have to be admitted as real. The principal result is the detection of double periodicity with amplitudes of $\sim 0.05 \mathrm{mag}$. The rough estimates give $13.6 \pm$ $\pm 0.2 \mathrm{~min}$ and $10.20 \pm 0.05 \mathrm{~min}$ as the periods. Both the periods were observed on different nights but were not seen together.

Lasker and Hesser (1971) have called attention to remarkable similarity in the variable white dwarfs. Table I contains more recent data on these stars, and one can see that the similarity is revealed even better now. In particular, the similar colour indices and the multiple periodicities with a narrow range in period ratios are the typical features of the stars in Table I. These white dwarfs apparently form a group of

TABLE I

Properties of the variable white dwarfs

\begin{tabular}{lccccccll}
\hline Star & EG & $V$ & $B-V$ & $U-B$ & Sp & $\begin{array}{l}\text { periods } \\
(\mathrm{sec})\end{array}$ & $\begin{array}{l}\text { period } \\
\text { ratio }\end{array}$ & reference \\
\hline R 548 & 10 & 14.1 & +0.20 & -0.54 & DA & 273 & & $\begin{array}{l}\text { Lasker and Hesser } \\
(1971)\end{array}$ \\
G 44-32 & 72 & 16.6 & +0.29 & -0.58 & DC & $\begin{array}{r}213 \\
1638\end{array}$ & 1.28 & $\begin{array}{l}\text { Lasker and Hesser } \\
\text { (1969) }\end{array}$ \\
& & & & & & 600 & 1.37 & Shulov and Kopatskaya \\
G 29-38 & 159 & 13.1 & +0.20 & -0.65 & DA & 816 & & $\begin{array}{l}812 \\
\text { (1974a, b) }\end{array}$ \\
HL Tau-76 & 265 & 15.2 & +0.20 & -0.50 & DA & $\begin{array}{l}746 \\
794\end{array}$ & 1.33 & Fitch (1973) \\
& & & & & & 494 & 1.51 & \\
\hline
\end{tabular}


variable stars of a new type of which G 29-38 is the brightest member now and is a favourable object for detailed studies with high time resolution.

A probable cause of variability is the non-radial pulsation in the white dwarfs discussed recently by Osaki and Hansen (1973). The periods of $g_{1}$ - and $g_{2}$-modes estimated in that paper coincide within an order of magnitude with the observed periods if a convective model is the case for all the variable white dwarfs except $R 548$ (a radiative model may be the case for $\mathrm{R} 548$.) In addition, the ratio $P\left(g_{2}\right) / P\left(g_{1}\right)$ coincides well with the period ratios in Table $I$, an important feature which was not stressed by Osaki and Hansen. It is unknown why the white dwarfs pulsate.

\section{References}

Eggen, O. J. and Greenstein, J. L.: 1965, Astrophys. J. 141, 83.

Faulkner, J., Flannery, B. P., and Warner, B.: 1972, Astrophys. J. 175, L79.

Fitch, W. S.: 1973, Astrophys. J. 181, L95.

Lasker, B. M. and Hesser, J. E.: 1969, Astrophys. J. 158, L171.

Lasker, B. M. and Hesser, J. E.: 1971, Astrophys. J. 163, L89.

Osaki, Y. and Hansen, C. J.: 1973, Astrophys. J. 185, 277.

Shulov, O. S. and Kopatskaya, E. N.: 1974a, Astron. Tsirk. (U.S.S.R.) No. 810, 1.

Shulov, O. S. and Kopatskaya, E. N.: 1974b, Astrofizika (U.S.S.R.) 10, 117.

\section{DISCUSSION}

A. M. Cherepashchuk: What is the amplitude of the variability of the white dwarf G 29-38?

$O$.S. Shulov: The amplitude of regular brightness changes is $0 m 05$. The amplitude of irregular fluctuations is up to 0 ? 3 .

$V . F$. Shvartsman: Are there isolated flashes observed or smooth light variations?

O. S. Shulov: Irregular variations were observed. An interpretation, therefore, is difficult. More observations are needed. 